\title{
ASKyphoplan: a program for deformity planning in ankylosing spondylitis
}

\author{
Barend J. van Royen · Famke J. Scheerder • \\ Eric Jansen - Theo H. Smit
}

Received: 27 November 2006/Revised: 23 January 2007/Accepted: 29 March 2007/Published online: 18 April 2007

(C) Springer-Verlag 2007

\begin{abstract}
A closing wedge osteotomy of the lumbar spine may be considered to correct posture and spinal balance in progressive thoracolumbar kyphotic deformity caused by ankylosing spondylitis (AS). Adequate deformity planning is essential for reliable prediction of the effect of surgical correction of the spine on the sagittal balance and horizontal gaze of the patient. The effect of a spinal osteotomy on the horizontal gaze is equal to the osteotomy angle. However, the effect of a spinal osteotomy on the sagittal balance depends on both the correction angle and the level of osteotomy simultaneously. The relation between the correction angle, the level of osteotomy and the sagittal balance of the spine can be expressed by a mathematical equation. However, this mathematical equation is not easily used in daily practice. We present the computer program ASKyphoplan that analyses and visualizes the planning procedure for sagittal plane corrective osteotomies of the spine in AS. The relationship between the planned correction angle, level of osteotomy and sagittal balance are coupled into the program. The steps taken during an ASKyphoplan run are outlined, and the clinical application is discussed. The application of the program is illustrated by the analysis of the data from a patient recently treated by a lumbar osteotomy in AS. The software
\end{abstract}

B. J. van Royen $(\bowtie) \cdot$ F. J. Scheerder .

T. H. Smit

Department of Orthopaedic Surgery,

VU University Medical Center, P.O. Box 7057,

1007, MB Amsterdam, The Netherlands

e-mail: bj.vanroyen@vumc.nl

E. Jansen - T. H. Smit

Department of Physics and Medical Technology,

VU University Medical Center,

Amsterdam, The Netherlands can be used free of charge on the internet at http:// www.stega.nl under the heading "research" in the menu.

Keywords Ankylosing spondylitis · Osteotomy · Spine $\cdot$ Computer program $\cdot$ Deformity planning

\section{Introduction}

In ankylosing spondylitis (AS), the spine becomes a rigid beam of bone from the occiput down to the sacrum. With few exceptions, AS leads to a rigid thoracolumbar kyphotic deformity (TLKD). Surgical correction of the TLKD by an extending osteotomy of the spine may be considered. The goal of such a surgical procedure is to restore both the patients' capacity to see the horizon, as well as the sagittal balance of the ankylosed spine $[2,5,6,8]$.

Preoperative planning of the effect of a lumbar osteotomy on the correction of the horizontal gaze and sagittal balance of the spine in patients with TLKD complicating AS is of utmost importance. The effect of a spinal osteotomy on the correction of the horizontal gaze is equal to the osteotomy angle, irrespective of the osteotomy level. Though the effect of a spinal osteotomy on the sagittal balance of the spine depends on both the osteotomy angle and the level of osteotomy simultaneously the correction of the sagittal balance is maximal when the intervention is performed at the lowest possible level of the lumbar spine [8].

Recently, a biomechanical and mathematical method for deformity planning for sagittal plane corrective osteotomies of the spine in AS has been developed. The effect of a lumbar osteotomy in AS on the horizontal gaze, defined as the chin-brow to vertical angle (CBVA) and the sagittal balance of the spine, defined as the sagittal vertical axis 
(SVA), can be expressed by a mathematical equation [6]. Following the promoted mathematical equation, a nomogram subsequently shows the relationship between the osteotomy angle, the level of osteotomy, and the sagittal balance. Unfortunately, both the crucial mathematic equations and the resulting nomogram are not easy to use in daily practice.

ASKyphoplan is a computational program that analyses and visualizes the planning procedure for sagittal plane corrective osteotomies of the lumbar spine in AS, which can be used easily in daily practice according to the promoted biomechanical and mathematical principles [6]. The purpose of the present work is to discuss the program and its performance. To this end, we illustrate the preoperative planning with ASKyphoplan on a patient, recently treated by a lumbar osteotomy in AS. The software can be used free of charge on the internet at http://www.stega.nl under the heading "research" in the menu.

\section{Description of an ASKyphoplan run}

The computational program ASKyphoplan is based on the biomechanical and mathematical principles described earlier [6]. In the present description of an ASKyphoplan run, we define the plumb line from the centre of the vertebral body of C7 and we plan the osteotomy at level L4. The normal sacral endplate angle (SEA) is defined at $40^{\circ}$ with the horizontal. Obviously, all variables can be changed independently for each case.

\section{Radiographic analysis}

A standard full-length lateral radiograph of the whole spine with the patient standing in relaxed standing position is made. Preferably, the radiograph is acquired and saved in a picture archiving and communication system (PACS). However, the use of a digitized regular film is also possible. The centre of body C7, the posterior superior corner of the sacrum (PSCS), the sacral endplate (SEA), and the rotation point (RP) on the anterior cortex of L4 are marked on the radiograph. In addition, the $50 \mathrm{~mm}$ grid of the film or two randomly chosen points are measured using the 'measuring distance' tool displayed on the digital system for calibration. Finally, the marked radiograph is saved in JPEG, Dicom or PNG format on a personal computer (Fig. 1).

\section{ASKyphoplan run}

Open the file with the saved radiograph in ASKyphoplan and upload the digitalized radiograph including the marks.
Five consecutive pages are followed: 'Calibration', 'Draw', 'Set SEA', 'Calculate', and 'Report'.

\section{Calibrate}

Mark the two measured reference points or the two marks on the grid of the film and define the distance in $\mathrm{mm}$ for calibration in the program.

\section{Draw}

The preceding marked reference points on the radiograph serve as guidance for drawing the deformity planning. The posterior superior corner of the sacrum (PSCS) is marked and a co-ordinate system appears. Then, the sacral endplate angle (SEA) from the posterior superior corner of the sacrum, parallel to the sacral endplate is drawn. The individual (compensated) SEA will appear in degrees. The centre of body $\mathrm{C} 7$ is marked, and the plumb line from $\mathrm{C} 7$ appears. The sagittal vertical axis (SVA), defined as the horizontal distance between the posterior corner of the sacrum and the plumb line from C7, will be shown. Finally, the aimed hinge or rotation point (RP) at the anterior cortex of L4 is marked.

\section{Set SEA}

The normal SEA is assumed to make an angle of approximately $40^{\circ}$ with the horizontal $[1,6]$. By defining the SEA at $40^{\circ}$, the radiograph will be projected onto the co-ordinate system with the SEA at $40^{\circ}$. The pre-operative SVA without compensation appears in millimeters.

\section{Calculate}

The relation between the correction angle, the level of osteotomy and the sagittal balance of the spine is calculated and visualized in the computer program. By planning the osteotomy at level L4 and aiming the post-operative SVA at $75 \mathrm{~mm}$ for example [6], the aimed correction angle at L4 and resulting correction of the CBVA follows. Using the 'visibility' bar, the virtual post-operative radiograph can be visualized (Fig. 3). The effect of changing the level of osteotomy on the SVA and CBVA correction or the effect of changing the correction angle on the SVA and CBVA can be visualized clearly by changing these variables in the program.

\section{Report}

The preoperative deformity planning, calculation and visualization of the osteotomy can be saved as a PDF file and printed for documentation. 


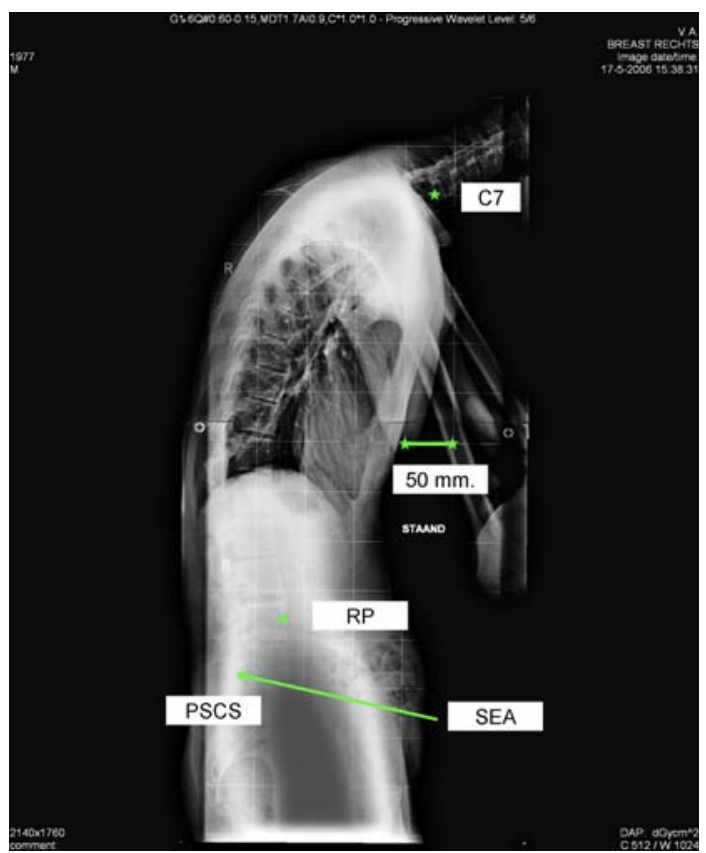

Fig. 1 The steps involved in radiographic analysis. A standard fulllength lateral radiograph of the whole spine with the patient standing in relaxed standing position is made. C7 the centre of body C7, PSCS the posterior superior corner of the sacrum, SEA the sacral endplate, $R P$ the rotation point on the anterior cortex of L4 are marked on the radiograph. In addition, the $50 \mathrm{~mm}$ grid of the film or two randomly chosen points are measured using 'measuring distance' tool displayed on the digital system for calibration

\section{Case report}

A 29 year-old man with a 8 year history of AS and a progressive TLKD is indicated for lumbar osteotomy
(Fig. 2a). The standard full-length lateral radiograph of the whole spine showed a pre-operative SEA of $14^{\circ}$. Rotation of the radiograph onto the co-ordinate system with the SEA at $40^{\circ}$ resulted in a pre-operative SVA of $384 \mathrm{~mm}$ (Fig. 3). Planning a SVA $75 \mathrm{~mm}$ anterior to the posterior superior corner of the sacral endplate, a correction angle of $43^{\circ}$ at level L4 is required. In addition, a $43^{\circ}$ correction at L 4 will correct the CBVA with $17^{\circ}$ (Fig. 3). The surgical procedure, including positioning of the patient, anaesthesia and surgical technique was performed according to the technique described earlier [8]. During the closure procedure of the closing wedge osteotomy, lateral images of the lumbar spine from the image intensifier were sent wireless to the local PACS at regular times. The acquired correction was measured in degrees by measuring the Cobb angle on the acquired images between the upper and lower endplate of L4 regularly, using 'measuring angle' tool displayed on the digital system, until the required correction angle was achieved (Fig. 4a, b). The post-operative radiograph of the lumbar spine showed a correction of $40^{\circ}$ in L4. There were no complications during surgery and follow-up treatment. The clinical postoperative CBVA showed to be 27 (Fig. 2b).

\section{Discussion}

Adequate deformity planning for sagittal plane corrective osteotomies of the spine in AS is essential for reliable prediction of the effect of surgical correction of the spine. ASKyphoplan is a computational program that analyses and visualizes the planning procedure for sagittal plane
Fig. 2 Clinical appearance $\mathbf{a}$ before and $\mathbf{b}$ after closing wedge osteotomy. The preoperative $\mathrm{CBVA}$ is $63^{\circ}$, and the postoperative $\mathrm{CBVA}$ is $27^{\circ}$
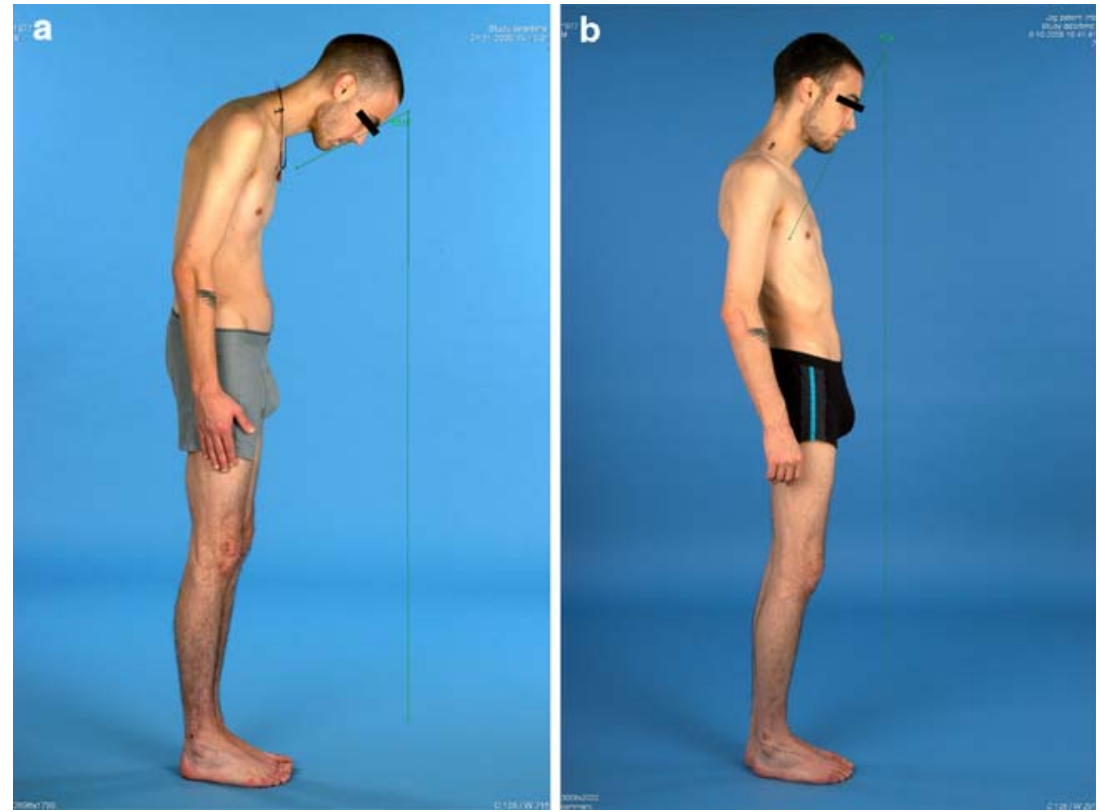


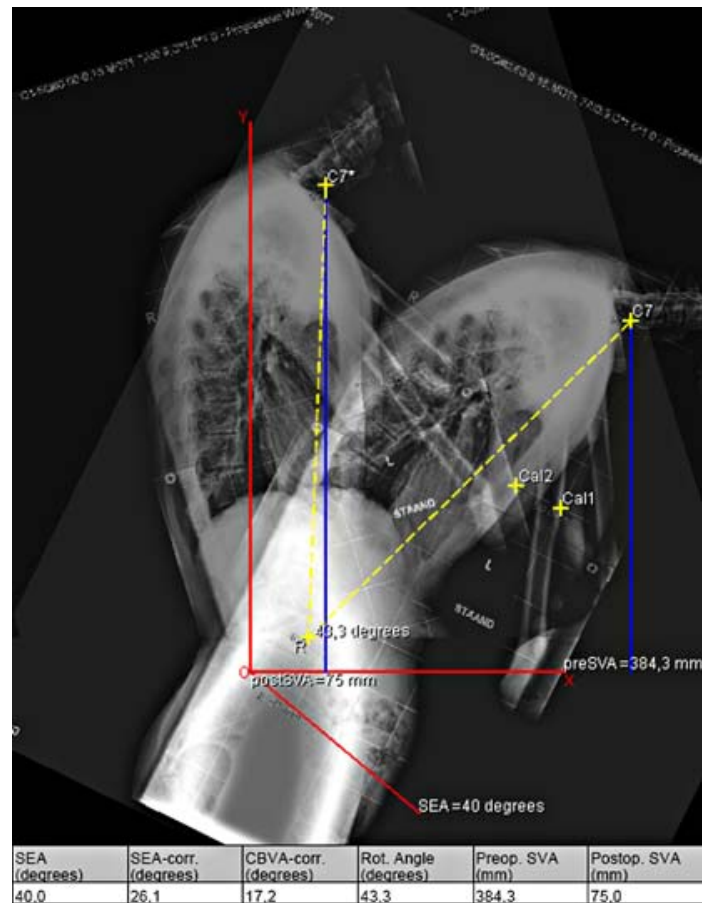

Fig. 3 ASKyphoplan report

corrective osteotomies of the lumbar spine in AS, which can be used easily in daily practice. The program analyses and visualizes the relationship between the osteotomy angle, the level of osteotomy in relation to the sagittal balance and CBVA for sagittal plane corrective osteotomies. In fact, deformity planning with ASKyphoplan can be performed for any patient with TLKD attributable to AS. All mentioned variables can be altered freely in the program for each individual patient and or by each individual surgeon.

ASKyphoplan professes not to represent the only, or necessarily the best, method for deformity planning in AS, but is rather meant as a method of the authors which may be helpful to others who treat patients with progressive TLKD caused by AS. The need for adequate deformity planning for sagittal plane corrective osteotomies of the spine in AS, however, is demonstrated by three recent papers [3, 4, 7]. Suk et al. [4] emphasized the clinical importance of the CBVA as an objective index for evaluating the horizontal gaze before and after lumbar osteotomy in AS. They reported on a series of 34 patients with AS treated with a closing wedge osteotomy. The osteotomy was performed at the apex of the kyphosis from Th7 to L2, and the mean correction angle was $37.7^{\circ}$. However, in seven cases $(21 \%)$ there was an overcorrection of the CBVA of more than $10^{\circ}$ hyperextension. Unfortunately, the authors did not report the correlation between the level of osteotomy, degree of correction, and CBVA correction. Deformity planning with ASKyphoplan will provide the
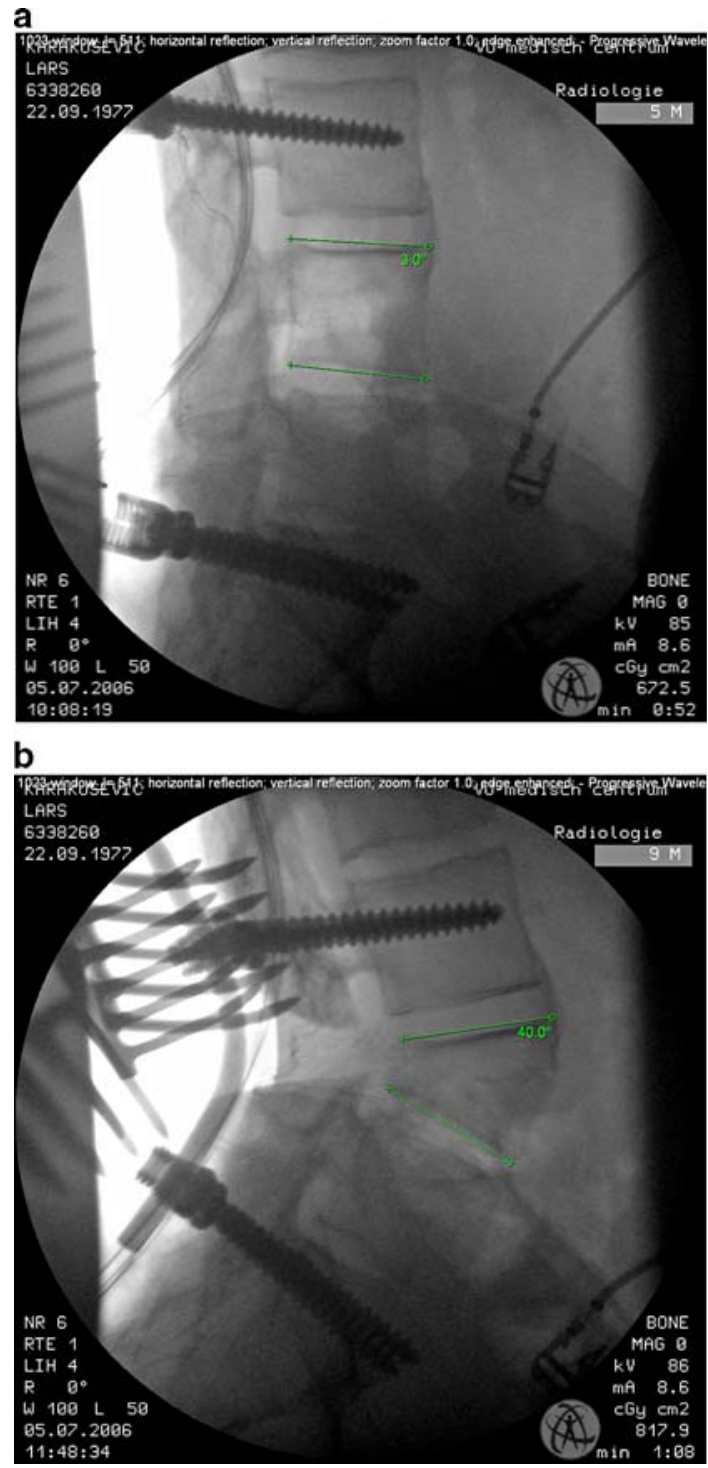

Fig. 4 During the closure procedure lateral images of the lumbar spine from the image intensifier were sent wireless to the local PACS at regular times (a). The correction is measured in degrees by measuring the Cobb angle on the acquired images between the upper and lower endplate of L4 regularly, until the required correction angle was achieved (b)

relationship between the correction angle, level of osteotomy, CBVA correction, and sagittal balance easily. Sengupta et al. [3] described the treatment of a case with a severe thoracolumbar kyphotic deformity resulting from AS in which a lordosing osteotomy of the mid-thoracic spine caused an extension deformity of the cervical spine. The authors described a flexion osteotomy of the cervical spine to correct the caused iatrogenic extension deformity. The reported case, a 44 year-old woman with a $120^{\circ}$ thoracic kyphosis, and a fixed cervical spine with a CBVA of $45^{\circ}$ due to AS, underwent a closing wedge osteotomy at T6 and T8. A total osteotomy angle of $75^{\circ}$ was achieved. 
As a result, an overcorrection of the CBVA of $30^{\circ}$ needed a corrective flexion osteotomy of the cervical spine. The case illustrates well the need for adequate deformity planning for sagittal plane corrective osteotomies of the spine in AS. Overcorrection of the CBVA and the subsequent need for a corrective cervical flexion osteotomy as described in this case can be prevented with the use of ASKyphoplan in future. Finally, this method of deformity planning is also essential in patients with a kyphotic Andersson lesion complicating AS [7]. In these cases the level of osteotomy is fixed by the location of the Andersson lesion. As expected, correction of the Andersson lesion in a high lumbar region will not result in the optimal restoration of the sagittal balance. However, overcorrection at the level of the Andersson lesion, in an attempt to achieve a better correction of the sagittal balance, may result in an extension deformity of the cervical spine forcing the visual field upward.

There are some clinical assumptions and technical difficulties in the procedure that can be criticized. Firstly, we defined the normal SEA to be $40^{\circ}$ to the horizon. In healthy subjects, the SEA makes an angle of approximately $40^{\circ}$ with the horizon on a standing lateral radiographic projection $[1,6]$. With the normal SEA of $40^{\circ}$, the hip joints are in the zero position, thus allowing for small compensatory movements of the pelvis. Obviously, alterations for the normal SEA can be applied individually. Secondly, the SVA is measured by calculating the horizontal distance between the posterior superior corner of the sacrum and the plumb line from C7. Unfortunately, C7 is not always that visible on the sagittal radiograph in patients with severe TLKD because of the over projection of the upper extremities. In individual cases the SVA can be defined as the horizontal distance between the posterior superior corner of the sacral endplate and the plumb line from any other cervical vertebra, for example the spinal process of $\mathrm{C} 7$ or vertebra $\mathrm{C} 2$. Thirdly, it should be realized that the optimum post-operative SVA in patients with TLKD resulting from AS is not known. We hypothesized that the post-operative SVA from C7 should be planned between 50 and $100 \mathrm{~mm}$ anteriorly of the posterior superior corner of the sacral endplate to prevent over correction of the ankylosed TLKD in AS [6]. For that reason, a postoperative SVA of $75 \mathrm{~mm}$ was planned in our case. However, further studies are necessary to evaluate the optimum postoperative SVA in patients with TLKD complicating AS. In addition, the relation between the measurements of the SVA from C7 and other measurement points e.g. the spinous process of $\mathrm{C} 7$ or the vertebra of $\mathrm{C} 2$ in deformity planning has to be analysed. Finally, the interobserver and intraobserver reliability of ASKyphoplan should be analysed in further studies.

In conclusion, ASKyphoplan is a computational program that analyses and visualizes the planning procedure for sagittal plane corrective osteotomies of the spine in AS. The relationship between the planned correction angle, level of osteotomy, CBVA correction, and sagittal balance are coupled into the program. The program can be used easily in daily practice.

\section{References}

1. Jackson RP, Peterson MD, McManus AC, Hales C (1998) Compensatory spinopelvic balance over the hip axis and better reliability in measuring lordosis to the pelvic radius on standing lateral radiographs of adult volunteers and patients. Spine 23:1750-1767

2. Kim KT, Suk KS, Cho YJ, Hong GP, Park BJ (2002) Clinical outcome results of pedicle subtraction osteotomy in ankylosing spondylitis with kyphotic deformity. Spine 27:612-618

3. Sengupta DK, Khazim R, Grevitt MP, Webb JK (2001) Flexion osteotomy of the cervical spine: a new technique for correction of iatrogenic extension deformity in ankylosing spondylitis. Spine 26:1068-1072

4. Suk KS, Kim KT, Lee SH, Kim JM (2003) Significance of chinbrow vertical angle in correction of kyphotic deformity of ankylosing spondylitis patients. Spine 28:2001-2005

5. Van Royen BJ, De Gast A (1999) Lumbar osteotomy for correction of thoracolumbar kyphotic deformity in ankylosing spondylitis. A structured review of three methods of treatment. Ann Rheum Dis 58:399-406

6. Van Royen BJ, De Gast A, Smit TH (2000) Deformity planning for sagittal plane corrective osteotomies of the spine in ankylosing spondylitis. Eur Spine J 9:492-498

7. Van Royen BJ, Kastelijns RC, Noske DP, Oner FC, Smit TH (2006) Transpedicular wedge resection osteotomy for the treatment of a kyphotic Andersson lesion complicating ankylosing spondylitis. Eur Spine J 15:246-252

8. Van Royen BJ, Slot GH (1995) Closing-wedge posterior osteotomy for ankylosing spondylitis. Partial corporectomy and transpedicular fixation in 22 cases. J Bone Joint Surg Br 77:117-121 